\title{
An Economic Solution to Optimize Performance of Photovoltaic Modules under Partial Shading
}

\author{
Paoyuan Lin ${ }^{1}$, Kok Chin Chai ${ }^{1}$, Herchang Ay ${ }^{1,2}$ \\ ${ }^{1}$ Department of Mold and Die Engineering, National Kaohsiung University of Applied Sciences, Taiwan \\ ${ }^{2}$ St. John's University, Taiwan
}

\begin{abstract}
This paper investigates the effects of partial shading pertaining to photovoltaic (PV) modules and subsequently a solution that aims to minimize the effects and optimize the performance of PV modules is proposed. The concept of the solution is to integrate boost and buck converters into an electronic control system. The proposed system is simple and economic solution as it integrates cheap and easy available electronic components such as microcontrollers, capacitors, transistors and inductors. To validate the effectiveness of proposed system, a measuring instrument, namely NI cFP-1808 is used to perform the electrical characteristic analyses or known as I-V and P-V curve analyses. Furthermore, an outdoor experiment was conducted to examine the practicality of the proposed system in tackling real-world conditions. Experimental results showed that the proposed system improves the performance of the PV modules by $82.9 \%$ compared to the controlled experiment. In short, the effectiveness and practicality of the proposed system is proven with experiment results.
\end{abstract}

\section{Introduction}

Photovoltaic (PV) system has received prominent attention over the last decade as it offers great advantages such as low maintenance cost, absence of moving or rotating parts and freedom from environmental pollution $[1,2]$. Though the PV system has been widely implemented, there are still numbers of ongoing limitations that need to be investigated and improved. As an example, partial shading on PV system is one of the ongoing issue $[3,4]$. Partial shading is defined as the PV arrays that are partially shadowed by the neighboring trees, buildings, towers and even passing clouds [3]. Under partial shaded conditions, the PV characteristics get more complex with multiple peaks. A research from Germany shows that the effect of partial shading can reduce the output energy up to $20 \%$ [4].

Literature reveals that numbers of researches have been conducted to tackle partial shading conditions. These researches can be categorized into maximum power-point tracking (MPPT) methods [2], modeling and simulation based approaches $[3,5,6]$, DC-DC converter [7]. In [2], a metaheuristic optimization algorithm namely, firefly algorithm, is used to track the maximum power point under partial shading condition in a PV system. A comprehensive study on MPPT methods has been discussed in $[8,9]$. In [3, 5], MATLAB-based modeling and simulation approach studies the effect of shading patterns on PV panels pertaining to different configurations. In [6], PSpice-based modeling and simulation approach investigates the effects of bypass diode configurations on PV modules. In [7], a DC-DC converter is introduced to boost the output energy in partial shading condition. While many solutions pertaining to partial shading are available, an economic and simple solution is relatively new.

The aim of this paper is to propose an electronic system that improves the performance of photovoltaic modules under partial shading. It is worth-mentioning that the proposed system is built with cheap and easily available components such as microcontrollers, capacitors, transistors and inductors. The system consists of two parts namely, boost converter and buck converter. In general, the boost and buck converters are used to increase and decrease the output voltage level pertaining to input voltage, respectively. The performance of the proposed system is validated using a measuring instrument, namely NI cFP-1808. NI cFP-1808 is designed to measure electric current, voltage and power. Besides that, it can also perform the electrical characteristic analyses or known as I-V and P-V curve analyses with simple configurations.

In this paper, the partial shading effect is investigated through different shading portions. To effectively model the shading portion, an opaque tape with good thermal insulation is used to shade the PV cell. The materials used to shade the PV cell should be carefully considered else it may collect heat that will indirectly affect the performance of PV cell. The effectiveness and practicality of the proposed system is further evaluated with an outdoor experiment. 
The rest of the paper is organized as follows. Section 2 introduces the notations with respective descriptions and some important equations. Section 3 presents the proposed system. Section 4 presents the experiment setup pertaining to two different investigations. Section 5 discusses the experiment results from different investigations. Finally, Section 6 presents the conclusion and future works.

\section{Preliminaries}

The related notations and their descriptions are listed in Table 1 as follows. As an example, $V_{\text {oc }}$ represents open circuit voltage.

Table 1. List of notations

\begin{tabular}{cl}
\hline Notations & Descriptions \\
\hline$V_{O C}$ & Open circuit voltage \\
$I_{s C}$ & Short circuit current \\
$V_{p m}$ & Maximum power voltage \\
$I_{p m}$ & Maximum power current \\
$F F$ & Fill factor \\
$P_{\max }$ & Maximum power \\
$P_{i n}$ & Input power \\
$\eta$ & Efficiency of photovoltaic $(\mathrm{PV})$ cell \\
$\Delta I_{s c}$ & Declined percentage for $I_{\mathrm{sc}}$ \\
$\Delta I_{p m}$ & Declined percentage for $I_{p m}$ \\
$\Delta P_{\max }$ & Declined percentage for $P_{\max }$ \\
\hline
\end{tabular}

Besides several important definitions and equations used in this paper are defined as follows.

Fill factor, $F F$ is defined as the ratio of maximum obtainable power to the product of the open-circuit voltage and short-circuit current. FF can be calculated using Eq. (1).

$$
F F=\frac{I_{m p} \times V_{m p}}{I_{S C} \times V_{O C}}
$$

Maximum power, $P_{\max }$ is product of $V_{O C}, I_{S C}$ and $F F$ as simplified in Eq. (2).

$$
P_{\max }=V_{O C} I_{S C} F F
$$

The efficiency of a PV cell is an important parameter to compare the performance of PV cell. It is defined as the fraction of energy output from the PV cell to input energy from the sun which can be simplified into Eq. (3)

$$
\eta=\frac{P_{\text {max }}}{P_{\text {in }}}
$$

where the input power per meter square of PV cell is 1 $\mathrm{kW} / \mathrm{m}^{2}$.

\section{The proposed system}

The proposed system consists of two parts, i.e., buck converter and boost converter. Both converters are similar in design. The general concept of the converters is illustrated in the block diagram as shown Figure 1. It comprises three modules namely, voltage regulator, pulse width modulation (PWM) generator and inductor-based switching regulator (SR). The first module is used to maintain a constant voltage at $10 \mathrm{~V}$. The second module is known as PWM to generate $40 \mathrm{kHz}$ frequency and the third module is used to step up/down of voltage source.

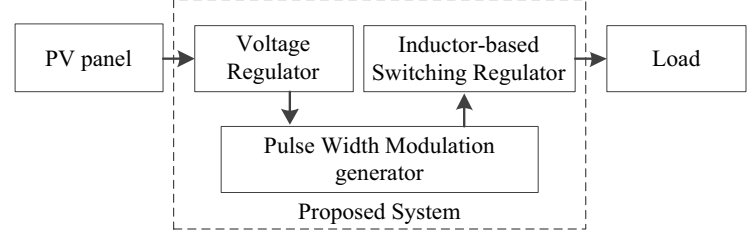

Figure 1. Block diagram of the proposed system

The three modules are integrated and designed in a single printed circuit board (PCB). An example of PCB layout for the proposed buck Converter is depicted in Figure 2.

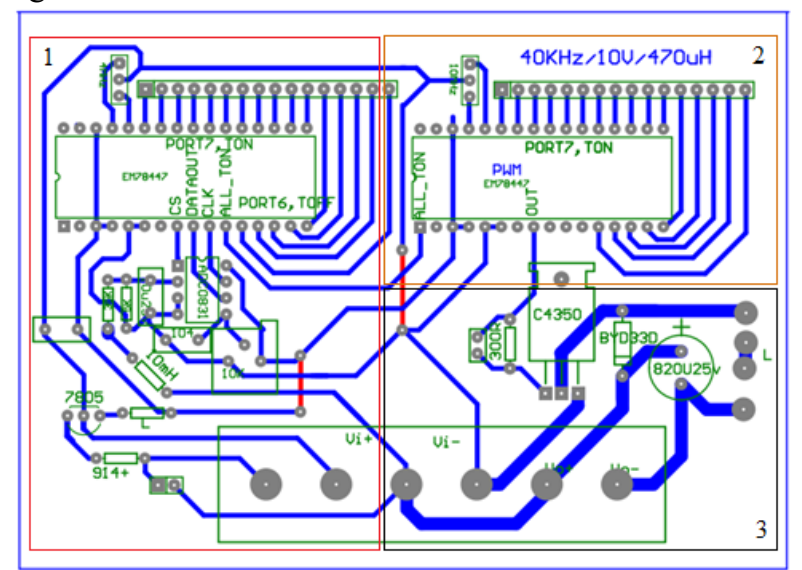

(a)

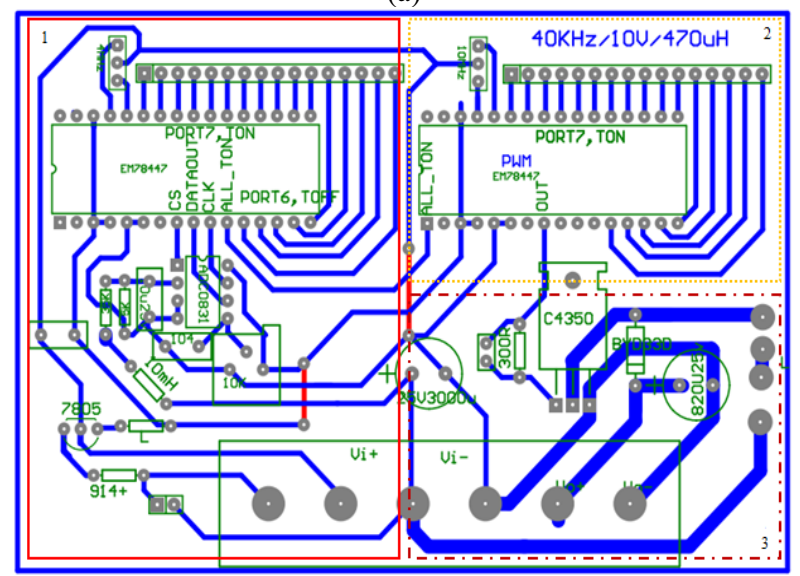

(b)

Figure 2. PCB layout diagram of the proposed (a) buck Converter (b) boost Converter

The buck converter consists of few electronic components such as two microcontrollers, EM78447, two crystal oscillators (i.e., $4 \mathrm{MHz}$ and $10 \mathrm{MHz}$ ), Analog to Digital convertor (ADC), capacitors, transistors, inductors and etc. As in Figure 2, it is observed that the square outline labeled with " 1 " indicates the voltage regulator module, " 2 " indicates the PWM generator module and " 3 " indicates the inductor-based SR module. In general, the circuit connection for both converters is 
similar, except for the configuration in third module. The difference for boost and buck inductor-based SRs is indicated in Figure 3. Technically, the buck inductorbased SR reduces a DC voltage to a lower DC voltage and boost inductor-based SR provides an output voltage that is higher than the input. With these boost and buck inductor-based SR, voltages can be regulated consistently. It is worth-mentioning that the inductor-based SR is beneficial as it has minimal power loss and provides high power efficiency [10].
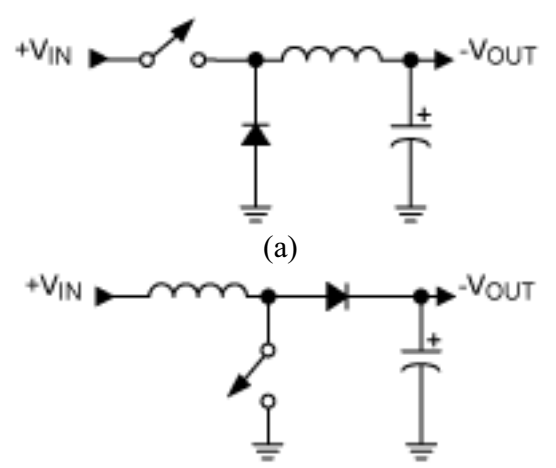

(b)

Figure 3. Inductor-based switching regulators (a) buck and (b) boost [11]

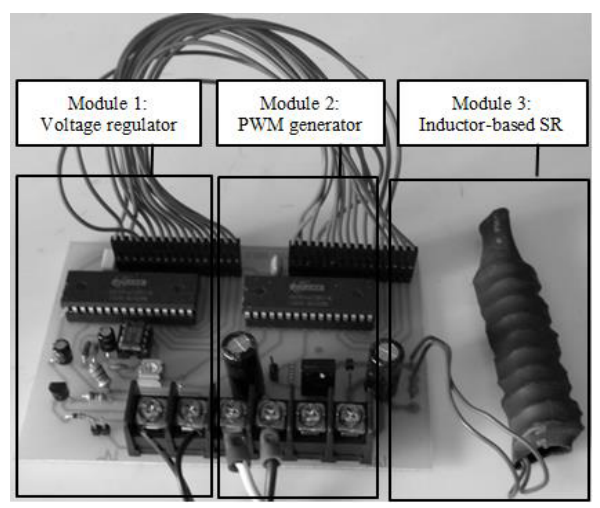

Figure 4. The proposed buck converter

\section{Experiment setup}

In this experiment, a PV cell is an electrical device with dimension $19.5 \times 52 \mathrm{~mm}$ that converts the light energy into electricity energy. Therefore, the total power generated from a $\mathrm{PV}$ cell per meter square is $1.014 \mathrm{~W}$. A PV module is a packaged, connected assembly via the arrangement of $5 \times 4 \mathrm{PV}$ cells i.e., 5 cells in row and 4 cells in column as illustrated in Fig.5 (a). The total area of a PV module that occupy with PV cell is $20 \times 19.5 \times 52 \mathrm{~mm}$ or $20280 \mathrm{~mm}^{2}$. Total power generated from a PV module is $20.28 \mathrm{~W}$. A PV array is defined as numbers of PV modules connected in serial/parallel. This research used a notation "SxPy XXX" to explain the connection of PV array where $\mathrm{S}$ and $\mathrm{P}$ indicate serial and parallel connections, respectively while $\mathrm{x}$ and $\mathrm{y}$ indicates the number of $\mathrm{PV}$ modules that connect in either serial or parallel connection and XXX indicates the shaded portion. For examples, "S1P1 6.25 " represents a single PV module with $6.25 \%$ shaded portion and "S3P1 25" represents three PV modules connected in serial with $25 \%$ shaded portion.

It is worth-mentioning that our experiment focuses on two investigations, (1) investigate the effects of different shaded portions pertaining to a PV module and (2) investigate the practicality of the proposed method in improving the performance of PV module under partial shading effects. These two investigations are presented in detail as follow.

\subsection{Investigate the effects of different shaded portions}

A PV module pertaining to different shaded portions (i.e., $6.25 \%, 12.5 \%, 18.75 \%$ and $25 \%$ ) as shown in Figure 5 , is investigated under a controlled experiment. The performance of the PV module is examined by using a photovoltaic module tester (SPI-SUN SIMULATOR 350i). SPI-SUN SIMULATOR 350i detects the efficiency of PV module. The tester uses 1.3M Xenon lights to test PV module with dimension $2200 \mathrm{~mm} \times$ $1200 \mathrm{~mm}$. Technically, its requirements can meet with ASTM E927 Class A rating which is a standard specification for solar simulation used for photovoltaic testing. SPI-SUN SIMULATOR 350i measures the important parameters of PV module such as $V_{o c}, I_{\mathrm{sc}}, V_{\mathrm{pm}}$, $I_{\mathrm{pm}}, P_{\mathrm{max}}, F F, \eta, \Delta I_{\mathrm{sc}}, \Delta I_{\mathrm{pm}}, \Delta P_{\mathrm{max}}$. Also it provides important visualization i.e., I-V and P-V characteristic curves. The results and discussions from this experiment are further discussed in Section 5.1.
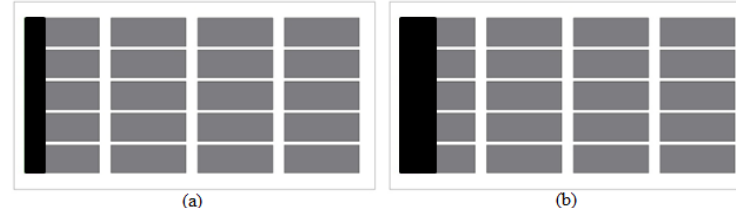

(a)
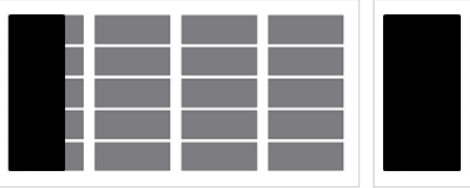

(b)

(c)
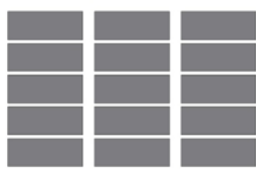

(d)

Figure 5. A PV module with different shaded portions (a) $6.25 \%$ (b) $12.5 \%$ (c) $18.75 \%$ (d) $25 \%$

\subsection{Investigate the proposed system pertaining to shaded portion}

To validate the practicality of the proposed system, an outdoor experiment is essential. The experiment was conducted on a sunny day from 0540 until 1840, at rooftop of Mold and Die Department, National Kaohsiung University of Applied Science, Taiwan. The setup of the experiment is shown in Figure 6. It consists of two groups of experiment, namely experiment and control groups. Both groups use three PV modules connected in serial (i.e., S3P1, in short). One of the three PV modules is shaded with $18.75 \%$, i.e., a total of $6.25 \%$ shaded portion for a PV array connected in S3P1. Besides that, both groups are controlled and applied that the same PV material and configurations, i.e., monocrystalline silicon, tilt angle $23.5^{\circ}$ and azimuth angle $175^{\circ}$ with $5^{\circ}$ facing south east.

The results from the experiment and control groups are collected using data acquisition system (i.e., National Instruments, NI cFP-1808). NI cFP-1808 has Input and 
Output (I/O) interface for Ethernet and RS232 networks. It can be easily read via the networked host PC. On the other hand, the solar irradiance of this experiment is measured using solar power meter (TEES-1333R) as depicted in left-corner of Figure 6.

Subsequently, the results from both groups are analyzed and compared. In this way, the practicality of the proposed system pertaining to shading effect of PV is validated. The results and discussions from the experiment are further discussed in Section 5.2.

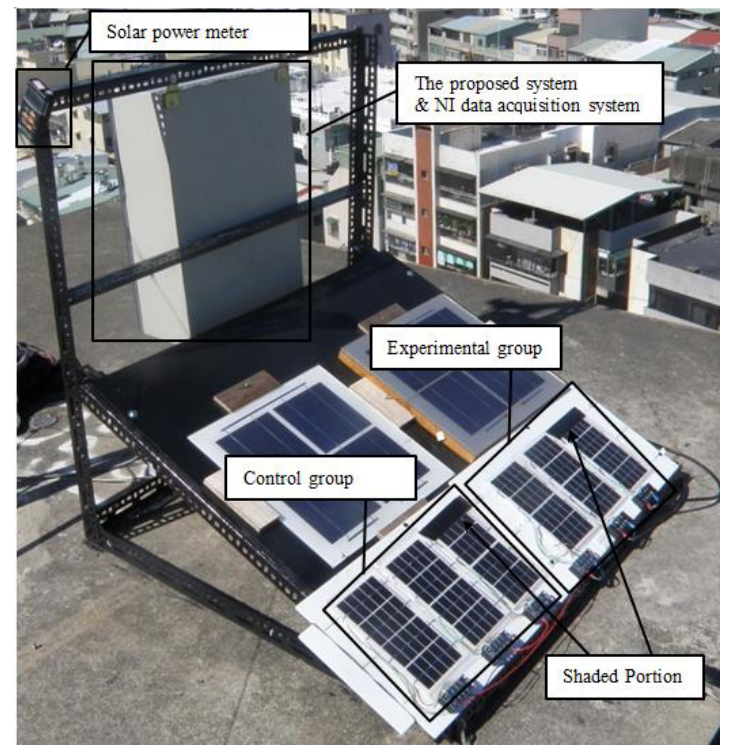

Figure 6. Setup for outdoor experiment

\section{Results and discussions}

The experimental results of the two investigations are presented in two sub-sections as follows.

\subsection{Results pertaining to different shaded portions}

Through SPI-SUN SIMULATOR 350i, PV without shaded portion obtains $V_{o c}$ is $12.35 \mathrm{~V}, I_{\mathrm{sc}}$ is $350.8 \mathrm{~mA}, V_{p m}$ is $10.25 \mathrm{~V}, I_{\mathrm{pm}}$ is $327.9 \mathrm{~mA}, P_{\max }$ is $3.362 \mathrm{~W}, F F$ is 0.776 and $\eta$ is $16.58 \%$. While the rest of the results for $6.25 \%$, $12.5 \%, 18.75 \%, 25 \%$ are tabulated in Table 2 . It is obvious that the efficiency of PV cell decreases drastically with the increase of shaded portions as in row " $\eta$ " it is observed that efficiency drop from $16.58 \%$ to
$13.18 \%, 8.955 \%, 4.655 \%$ and $0 \%$ for shaded portions of $6.25 \%, 12.5 \%, 18.75 \%, 25 \%$, respectively. Also, it is observed that the declined percentages pertaining to $I_{p m}$ i.e., $\Delta I_{p m}$ are increasing with respective to increase in shaded portions.

$\mathrm{I}-\mathrm{V}$ and $\mathrm{P}-\mathrm{V}$ characteristic curves in Figure 7 further illustrate the effect of different shaded portions. Again, the effect is significant as the increasing of shaded portions will lead to reduce in both current (A) and power (W), correspondingly. When the shaded portion is up to $25 \%$, it is observed that the power generated is reduced drastically to about $0 \mathrm{~W}$.

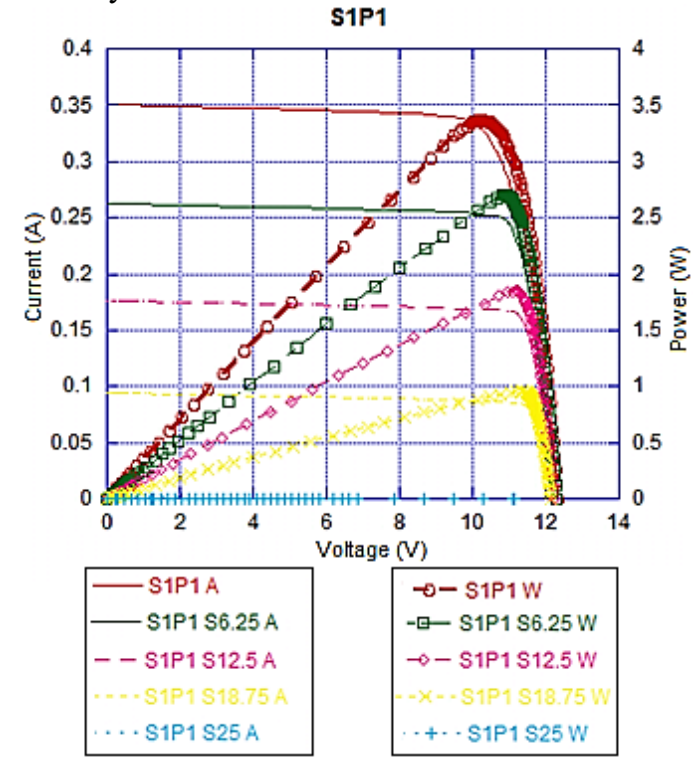

Figure 7. I-V and P-V characteristic curve of a PV module tested under different shading percentages

Further to this, other configurations such as S2P1 and S3P1 are investigated. The results are tabulated in Table 2. The results obtained from $\mathrm{S} 2 \mathrm{P} 1$ and $\mathrm{S} 3 \mathrm{P} 1$ are in line with the observations in S1P1. It is observed that the efficiency of PV cell reduces with the increase of shaded portions. It indicates that the increase of shaded portions significantly reduce the efficiency of PV module. Such deduction is also applicable to different configuration of PV module. As such, a research that improves the performance of the PV modules under partial shading is important.

Table 2. Experimental results of different PV configurations pertaining to different shading percentages

\begin{tabular}{|c|c|c|c|c|c|c|c|c|c|c|c|c|c|c|c|}
\hline \multirow{2}{*}{ Parameters } & \multicolumn{5}{|c|}{ S1P1 with different shading percentages } & \multicolumn{5}{|c|}{ S2P1 with different shading percentages } & \multicolumn{5}{|c|}{ S3P1 with different shading percentages } \\
\hline & $0 \%$ & $6.25 \%$ & $12.5 \%$ & $18.75 \%$ & $25 \%$ & $0 \%$ & $6.25 \%$ & $12.5 \%$ & $18.75 \%$ & $25 \%$ & $0 \%$ & $6.25 \%$ & $12.5 \%$ & $18.75 \%$ & $25 \%$ \\
\hline$I_{s c}$ & 350.8 & 262.5 & 176.7 & 94.40 & 1.100 & 350.7 & 351.6 & 351.0 & 350.8 & 350.9 & 350.9 & 350.8 & 351.0 & 350.9 & 350.7 \\
\hline$I_{p m}$ & 327.9 & 238.1 & 160.4 & 85.00 & 0.600 & 20.52 & 20.80 & 51 & 9.393 & 9.331 & 30.76 & 31.61 & 69 & 19.60 & 19.81 \\
\hline$P_{\max }$ & 3.362 & 2.673 & 1.816 & 0.944 & 0.004 & 324.6 & 254.5 & 180.7 & 323.4 & 323.4 & 325.1 & 259.0 & 323.5 & 324.0 & 323.3 \\
\hline$F F$ & 77.62 & 82.78 & 83.98 & 82.46 & 27.55 & 76.73 & 60.89 & 42.75 & 29.09 & 49.13 & 76.72 & 62.90 & 48.93 & 48.60 & 60.83 \\
\hline$\Delta I_{s c}$ & 0 & 25.17 & 49.63 & 73.09 & 99.69 & 0 & -0.257 & -0.086 & -0.029 & -0.057 & 0 & 0.029 & -0.029 & 0 & 0.057 \\
\hline$\Delta I_{p m}$ & 0 & 32.13 & 51.08 & 74.08 & 99.82 & 0 & 21.60 & 44.33 & -0.370 & -0.37 & 0 & 20.33 & 0.492 & 0.338 & 0.554 \\
\hline$\Delta P_{\max }$ & 0 & 20.49 & 45.99 & 71.92 & 99.88 & 0 & 20.55 & 44.36 & 54.41 & 54.71 & 0 & 18.15 & 36.32 & 36.53 & 36.60 \\
\hline
\end{tabular}




\subsection{Results pertaining to outdoor experiment}

The results of the outdoor experiment are summarized in Table 3. The maximum solar irradiance of the day is 960 $\mathrm{Wh} / \mathrm{m}^{2}$ at time 12:22:58. The maximum power collected through the experiment and control groups are $5.089 \mathrm{~W}$ and $2.742 \mathrm{~W}$, respectively. The maximum power collected through the experiment group is higher than the control group. This is the first evidence that the proposed method installed in experiment group has improved the power efficiency of PV modules under partial shading.

Further to this, the cumulative total generated power from the experiment group is $30.0 \mathrm{Wh}$ compare to the control group $16.4 \mathrm{Wh}$. It shows that the proposed system improved the total accumulated power by $13.6 \mathrm{Wh}$, i.e., the overall power efficiency improves by $82.9 \%$. Besides that, the performance of both groups is visualized in Figure 8. The cumulative total generated power of the experiment group is always higher than the control group throughout entire the experiment.

Table 3 Results of outdoor experiment

\begin{tabular}{ll}
\hline Details & Results \\
\hline $\begin{array}{l}\text { Duration of experiment } \\
\text { Maximum solar irradiance }\end{array}$ & $05: 40: 00 \sim 18: 30: 00$ \\
$\begin{array}{l}\text { Maximum power collected through the } \\
\text { experiment group }\end{array}$ & $560 \mathrm{Wh} / \mathrm{m}^{2}(12: 22: 58)$ \\
$\begin{array}{l}\text { Maximum power collected through the } \\
\text { control group }\end{array}$ & $2.742 \mathrm{Wh}(12: 22: 58)$ \\
$\begin{array}{l}\text { Cumulative total solar irradiance } \\
\text { Cumulative total generated power from } \\
\text { experiment group }\end{array}$ & $4921 \mathrm{Wh} / \mathrm{m}^{2}$ \\
$\begin{array}{l}\text { Cumulative total generated power from } \\
\text { control group }\end{array}$ & $16.0 \mathrm{Wh}$ \\
$\begin{array}{l}\text { Percentage of power restored via the } \\
\text { proposed system }\end{array}$ & $\mathbf{8 2 . 9 \%}$ \\
\hline
\end{tabular}

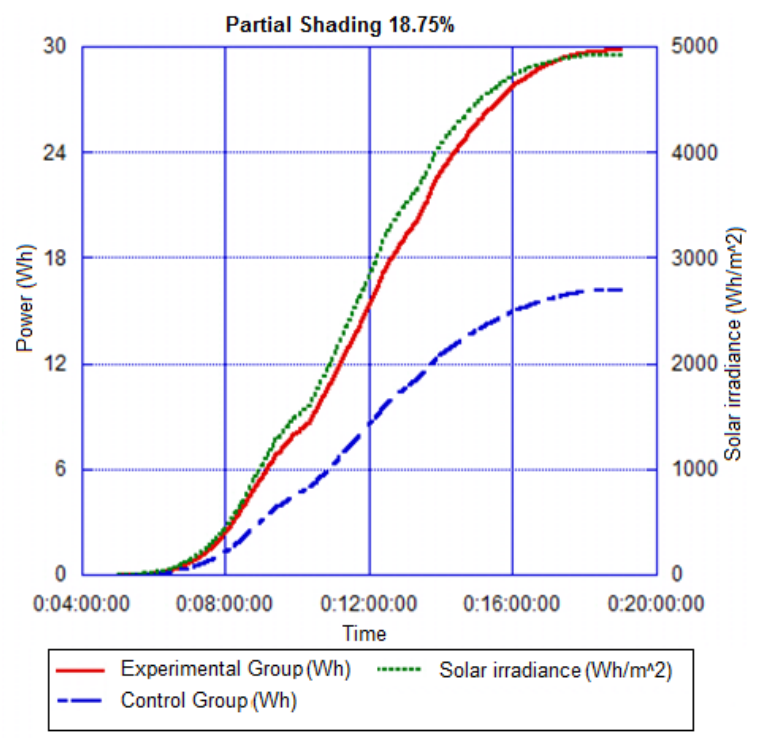

Figure 8. Cumulative generated power and solar irradiance

\section{Conclusion and future works}

In this research, the effects of different shading portions are investigated. The results show that the performance of PV cell is significantly affected. As such, a new solution that optimizes performance of photovoltaic modules under partial shading is proposed. Through the experiment, the proposed system has proven to improve the performance of the PV modules by $82.9 \%$. It is worth-noting that the proposed solution is important as it is simple and economic.

In future, it is important to further investigate the practicality of the proposed system pertaining to PV array. It is interesting to investigate the durability of the proposed system in real implementation.

\section{References}

[1] M. Z. S. EL-Dein, M. Kazerani, and M. M. A. Salama, "Optimal photovoltaic array reconfiguration to reduce partial shading losses," IEEE Trans. Sustain. Energy 4, 1 ( 2013)

[2] K. Sundareswaran, S. Peddapati and S. Palani, "MPPT of PV systems under partial shaded conditions through a colony of flashing fireflies", IEEE Trans. on Energy Convers. 29, 2,(2014)

[3] H. Patel and V. Agarwal, "MATLAB-Based Modeling to Study the Effects of Partial Shading on PV Array Characteristics", IEEE Trans. on Energy Convers. 23, 1 (2008)

[4] U. Jahn and W. Nasse, "Operational performance of gridconnected PV systems on buildings in germany", Prog. Photovolt: Res. Appl. 12, 6 (2004)

[5] K. Ishaque and Z. Salam,. "A comprehensive MATLAB Simulink PV system simulator with partial shading capability based on twodiode model", Sol. Energy 85, 9 (2011)

[6] S. Silvestre, A. Boronat, A. Chouder, "Study of bypass diodes configuration on PV modules", Appl. Energy 86 (2009)

[7] S. Strache et al., "Photovoltaic output power improvement applying DC-DC converters on submodule level," in Proc. IEEE Int. Conf. Smart Grid Technol. Economics Policies (2012)

[8] A. Bidram, A. Davoudi and R. S. Balog, "Control and circuit techniques to mitigate partial shading effects in photovoltaic arrays". IEEE J. Photovolt. 2, 4 (2012)

[9] B. Subudhi and R. Pradhan, "A comparative study on maximum power point tracking techniques for photovoltaic power systems," IEEE Trans. Sustain. Energy, 4, 1 (2013)

[10] A. Wang and S. Naffziger, Adaptive Techniques for Dynamic Processor Optimization: Theory and Practice. Springer Science \& Business Media (2008)

[11] "DC-DC Converter Tutorial - Tutorial Maxim". Maximintegrated.com. (2016) 